\title{
Computer-aided King classification of scoliosis
}

\author{
Junhua Zhang ${ }^{\mathrm{a}, *}$, Hongjian $\mathrm{Li}^{\mathrm{b}}$, Liang Lv ${ }^{\mathrm{b}}$, Xinling Shi ${ }^{\mathrm{a}}$ and Yufeng Zhang ${ }^{\mathrm{a}}$ \\ ${ }^{a}$ Department of Electronic Engineering, Yunnan University, Kunming, Yunnan, China \\ ${ }^{\mathrm{b}}$ Department of Orthopedics, the First People's Hospital of Yunnan Province, Kunming, Yunnan, China
}

\begin{abstract}
.
OBJECTIVE: Surgical planning for scoliosis relies on the classification of spinal curve pattern. The widely used King classification is subject to human measurement and judgment errors. To reduce the variability of King classification, a computer-aided method is proposed and its reliability is evaluated in this study.

METHODS: At the first step, endplate inclination and position of each vertebra are measured by a computerized system on the posteroanterior radiograph. Based on these measurements, the apical and end vertebrae are identified and then the Cobb angles are calculated automatically. The system subsequently classifies the curve types automatically based on the computerized Cobb measurements, the vertebral inclination, and the analysis of the relationship between the center sacral vertical line, and the apical lumbar vertebra. Five observers participated in the experiments. The kappa statistic was used to evaluate the variability. RESULTS: Classifications of 105 scoliotic cases by the five observers showed that with the aid of our system, the average intraobserver and interobserver kappa values improved from 0.75 to 0.90 and from 0.66 to 0.86 , respectively.

CONCLUSION: Results indicate that the proposed computerized system can assist a surgeon with the King classification of scoliosis.
\end{abstract}

Keywords: Computer-aided diagnosis, King classification, radiograph, scoliosis

\section{Introduction}

Idiopathic scoliosis (IS) is a complex three-dimensional (3D) deformity of the spine without known causes [1]. Classification of scoliotic curve pattern is important for the preoperative surgical planning. By identifying the curve pattern of spinal deformity, segmental instrumentation is applied on the suggested levels of the scoliotic spine for the deformity corrections. Currently, the King classification [2] is one of the most widely-used classification methods for IS. It defines five curve types and an additional group called "miscellaneous" based on measurements of standing coronal radiographs, and includes measurements from lateral bending films. Since the King classification relies on the subjective curve measurement and the interpretation of classification criteria, its reliability is an issue to be concerned. The reported empirical studies on repeat classification by the King method showed poor reliability [3]4].

Recently, some studies have developed computer-aided methods to measure scoliosis curves or to classify spinal deformity on radiographs. Stokes et al. [5]6] developed a computer program that applies a rule-based algorithm to automatically identify the King types based on the Cobb angles calculated from

\footnotetext{
${ }^{*}$ Corresponding author: Junhua Zhang, Department of Electronic Engineering, Yunnan University, No. 2 Cuihu Road, Kunming 650091, Yunnan, China. Tel./Fax:+86 871 65031301; E-mail: jhzhang_2006@163.com.
} 
coordinates of vertebral landmarks on the posteroanterior (PA) radiograph. Their approach required manual identification of numerous landmarks (70 landmarks per radiograph). The inherent variability in landmark identification might result in unreliability of measurement and classification. Lin [7] implemented an artificial neural network to automatically identify the King types based on features extracted from a simplified 3D spine model by the total curvature analysis, which was different from the traditional way of measuring scoliotic curves. Poncet et al. [8] proposed a classification system that defined three patterns of scoliotic curves based on the spinal geometric torsion. Duong et al. [9] used a wavelet transform of the vertebrae centroids and a fuzzy clustering algorithm to group 3D spine shapes. Both methods were technically elegant and illustrated that $3 \mathrm{D}$ classifications were important. However, those methods are not considered very intuitive by physicians.

In our previous study [10], a computer-aided approach that automatically measuring vertebral inclination was developed to improve the reliability in the Cobb measurement. In this study, we propose a computer-aided King classification approach using the computerized Cobb measurement to improve the reliability of the King classification.

\section{Materials and methods}

PA radiographs taken from 105 scoliotic patients (91 female and 14 male, age $14.5 \pm 3.6$ years old) were used in this study. The selection criteria were (1) diagnosis of idiopathic scoliosis, (2) no prior spine surgery, (3) ages between 9 years-18 years old, (4) Cobb angle less than $90^{\circ}$, and (5) visibility of the pelvis and T1 to L5 vertebral levels on the PA radiographs. The exclusion criteria were patients who had other musculoskeletal or neurological disorders. The main curves of patients included in this study presented a mean Cobb angle value of $46^{\circ} \pm 19^{\circ}$ measured by an orthopedic surgeon. This study received ethics approval from the local ethic board.

In our previous study [10], a fuzzy Hough transform (FHT)-based approach was developed for the Cobb measurement of a spinal curve. In this study we used this approach to identify the inclination of each vertebral endplate for the automatic King classification. This technique is described in the following subsections.

\subsection{Measurement of vertebral endplate inclination}

Each radiograph containing vertebrae from T1 to L5 is normalized to a standard height of 1000 pixels. The user assigns the names of the most upper and lower thoracic vertebrae on the radiograph. From the most upper thoracic vertebra, the user selects vertebrae by mouse clicks at the vertebrae. Once the user clicks at a vertebra, an initial rectangle of $100 \times 80$ pixels is created and displayed. The user can adjust the rectangle to fit the vertebra by rotating, moving, magnifying, or minifying the rectangle. For example, Fig. 1 shows the selected rectangles on a PA radiograph. The user-assigned rectangles defined the region of interest (ROI) for the vertebrae.

For each ROI, the Canny edge detection is performed to obtain the required edge image for the FHT. Because the radiograph contains a complex background due to various types of artifacts, it is necessary to delete noises and artifacts. According to the user-assigned rectangle, an inner rectangle and an outer rectangle are defined in the algorithm automatically. The distance between the inner rectangle and the user-assigned rectangle is set as $1 / 6$ of the width of the user-assigned rectangle, and the distance between the outer rectangle and the user-assigned rectangle is set as 1/8 of the width of the user-assigned rectangle. Currently the ROI just covers the outer rectangle. The noises and artifacts inside of inner rectangle 




Fig. 1. ROI selection and landmark identification.

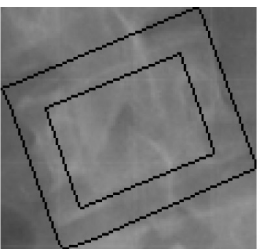

(a)

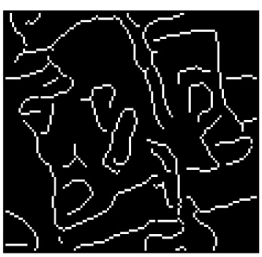

(b)

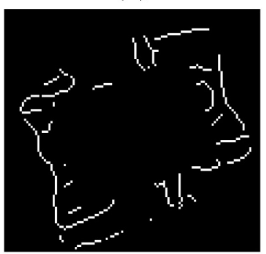

(c)

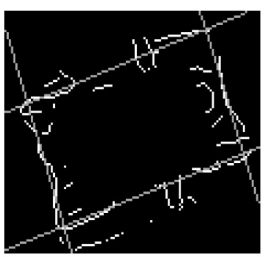

(d)

Fig. 2. Detection of endplates. (a) ROI with the inner and outer rectangles. (b) Edge image of the ROI. (c) Edge image with noises deleted. (d) Detected endplates.

and outside of outer rectangle are deleted from the ROI region. For example, Fig. 2 shows an ROI with the inner and outer rectangles (Fig. 2(a)), the edge image of the ROI (Fig. 2(b)), and the edge image with noises deleted (Fig. 2(c)). The FHT was performed separately on each edge image of the ROI.

In the Hough space, the vertebral shape priors are used to select the candidate peaks corresponding to the lines that best fitted to a vertebra (i.e., a pair of endplates and a pair of vertical edges): (1) the distance between two endplates of a vertebra is in the range of 30 pixels to 60 pixels, and the distance between two vertical edges is in the range of 40 to 80 pixels; (2) the average angle of two endplates is titled less than $45^{\circ}$, and the average angle of the vertical edges is between $45^{\circ}$ and $90^{\circ}$; (3) the angle difference between two endplates or two vertical edges of a vertebra is less than $10^{\circ}$; (4) the endplates and the vertical edges are close to perpendicular to each other. Using these shape priors, four lines fitting to a vertebra are identified. Figure 2(d) shows the detected lines for the edge image shown in Fig. 2(c). More details of this technique were described in our previous study [10].

The inclination of each line that fitted to vertebral endplate is recorded automatically. The inclination of a vertebra is calculated as the mean inclination of its two endplates. For each vertebra, the rectangle that consisted of four detected lines, i.e. the vertebral rectangle is also recorded. The position of the vertebra is defined as the center of the vertebral rectangle. 


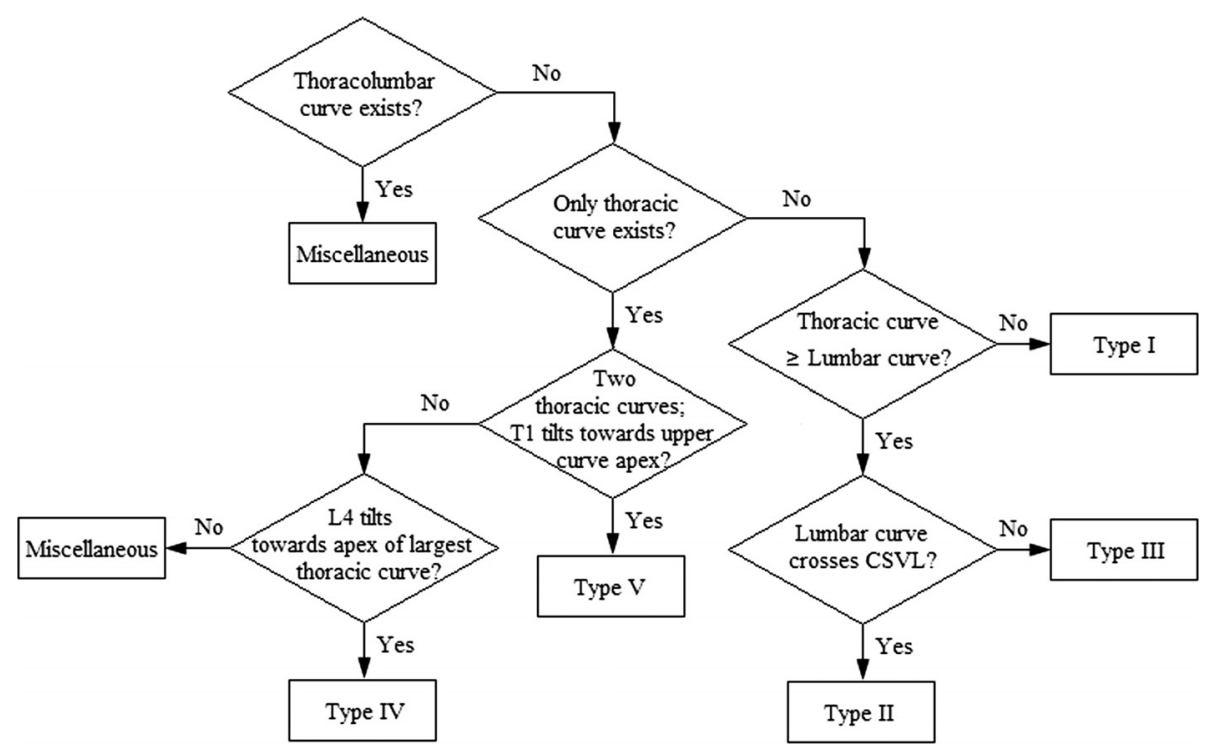

Fig. 3. Flowchart of the classification algorithm.

\subsection{Computer-aided King classification}

To implement the King classification, the central sacral vertical line (CSVL), the apical and end vertebrae, the Cobb angle, and the relationship between the CSVL and apical vertebra are determined. The user assigns two symmetrical landmarks on the sacrum of the PA radiograph by mouse clicks. According to the accepted definitions [5], the lateral edge of the radiograph is used to define the vertical direction. The CSVL is determined as the vertical line passing through the midpoint of the two sacral landmarks. The apical vertebrae is identified based on the detected endplate inclinations and vertebral positions. The apical region including the two vertebrae above, the two vertebrae below, and a disc separating two vertebrae tilting in opposite directions to the horizontal was first located. The vertebra in this region that had the greatest horizontal distance from the CSVL is identified as the apical vertebra. Based on the detected apical vertebra, the upper vertebra with the greatest inclination of the superior endplate and the lower vertebra with the greatest inclination of the inferior endplate are selected as the two end-vertebrae for a curve. The angle between the superior endplate of the upper end-vertebra and the inferior endplate of the lower end-vertebra is calculated as the Cobb angle automatically. Finally, the system decides whether an apical vertebra crossed the CSVL by identifying whether or not the CSVL touches the rectangle of the apical vertebra.

Based on the computer-aided Cobb measurement and identification of the apical vertebra, the computerized classification algorithm is used to implement the automatic King classification. The King classification defines five curve types. As analyzed by Stokes and Aronsson [5], the criteria of the original King classification are ambiguous. In this study, we used the criteria proposed by Stokes and Aronsson [5]. In Type I and II patterns, both the lumbar and thoracic curves cross the CSVL. The deformity is classified as Type I if the lumbar curve is larger than the thoracic curve, otherwise as Type II. Type III shows a thoracic curve where the lumbar curve does not cross the CSVL. There is no lumbar curve (the Cobb angle of $<10^{\circ}$ ) in Type IV and V patterns. Type IV shows a long thoracic curve where L4 tilts in the direction of the long thoracic curve. Type $\mathrm{V}$ shows a double thoracic curve pattern where $\mathrm{T} 1$ tilts into 
Table 1

Intraobserver reliability

\begin{tabular}{cccccc}
\hline \multirow{2}{*}{ Observer } & \multicolumn{2}{c}{ With aid } & & \multicolumn{2}{c}{ Without aid } \\
\cline { 2 - 3 } \cline { 5 - 6 } & Consistency & $\kappa$ & & Consistency & $\kappa$ \\
\hline 1 & $94 \%$ & 0.91 & & $81 \%$ & 0.76 \\
2 & $93 \%$ & 0.89 & & $81 \%$ & 0.74 \\
3 & $93 \%$ & 0.90 & & - & - \\
4 & $92 \%$ & 0.88 & & - & - \\
5 & $95 \%$ & 0.93 & & - & - \\
Average & $93 \%$ & 0.90 & & $81 \%$ & 0.75 \\
\hline
\end{tabular}

Table 2

Interobserver reliability

\begin{tabular}{cccccc}
\hline \multirow{2}{*}{ Trial } & \multicolumn{2}{c}{ With aid } & & \multicolumn{2}{c}{ Without aid } \\
\cline { 2 - 3 } \cline { 5 - 6 } & Consistency & $\kappa$ & & Consistency & $\kappa$ \\
\hline 1 & $85 \%$ & 0.82 & & $73 \%$ & 0.65 \\
2 & $87 \%$ & 0.88 & & $74 \%$ & 0.67 \\
3 & $93 \%$ & 0.90 & & $74 \%$ & 0.67 \\
Average & $88 \%$ & 0.86 & & $74 \%$ & 0.66 \\
\hline
\end{tabular}

the convexity of the upper curve. Following these criteria, the computerized classification algorithm is implemented, as shown in Fig. 3, to perform the automatic King classification,

\subsection{Evaluation}

In this study, five observers participated in the experiments, including a pediatric orthopedic surgeon with 10 years of experience in scoliosis clinic, an orthopedic resident, a musculoskeletal radiologist, a medical student without experience in orthopedic radiology, and the software developer without clinical experience. Each observer performed the tasks of identifying two symmetrical landmarks on the sacrum of the PA radiograph and setting the ROI for each vertebra three times over a period of three weeks. Without the aid of computer, the surgeon and the resident also individually classified spinal curve patterns three times using only the table description in the traditional King method [2] based on the Cobb measurement and apical vertebrae identification manually. The surgeon's first trial without the aid of computer classified 105 cases into 25 Type I, 21 Type II, 26 Type III, 29 Type IV, and 4 Type V cases.

The kappa statistic $(\kappa)$ in SPSS (SPSS Inc., Chicago, IL) was used to assess the reliability of the King classification under the conditions with and without the aid of computer. Under each of the two conditions, the $\kappa$ values were calculated for paired sets of classifications by each observer (intraobserver repeatability) or between observers (interobserver reliability), using all combinations of paired observations. The resulting average values were calculated over combinations of pairs (intraobserver or interobserver) to provide an overall assessment of reliability.

For the cases that were consistently classified by the surgeon three times without the computer aid, the surgeon's first trails with and without the computer aid were compared to evaluate accuracy of the proposed computerized system.

\section{Results}

Table 1 lists the intraobserver repeatability. With the aid of computer, the $\kappa$ values of five observers range from 0.88 to 0.93 , which are in the excellent range $(>0.80)$. By using the computerized method, the $\kappa$ values increase from 0.76 to 0.91 and from 0.74 to 0.89 for the surgeon and the resident, respectively. The average $\kappa$ value and the average classification consistency increase to 0.90 and $93 \%$, respectively.

Table 2 lists the interobserver reliability. By using the proposed method, the average interobserver $\kappa$ values increase from 0.66 to 0.86 and the average interobserver consistency increases from $74 \%$ to $88 \%$. Without the aid of computer, the overall interobserver $\kappa$ values increase from 0.65 to 0.67 over the three series of measurement, while with the computerized method, the $\kappa$ values increase from 0.82 to 0.90 . 
Without the aid of computer, the two observers in all three trials consistently classified 50 cases, while with the aid of computer, all five observers consistently classified 65 cases.

Without the aid of computer, the surgeon consistently classified 78 cases in three trials. For the 78 cases, the surgeon's first trials with and without the aid of computer were compared. Results showed that the surgeon's classifications were consistent in $95 \%$ cases (74 out of 78) under two conditions. Of the 4 cases that were classified inconsistently, three cases were due to inconsistent detection of the lumbar curve crossing the CSVL (Type II or III) and one case was due to inconsistent identification of an upper thoracic curve (Type V or IV).

The processing time was also evaluated. It took five minutes in total for each radiograph, and about three minutes after the user interaction.

\section{Discussion}

Consistency of curve type classification is important in the assessment and treatment of scoliosis. Poor reliability of the King classification was shown in previous studies [3|4]. Since it relies on subjective measurement and classification, there are high chance of intraobserver and interobserver variability. This study proposed a computer-aided method to improve the reliability of the King classification. The experimental results indicated that the tasks of measuring scoliosis curves on radiographs and subsequently classifying curve types were more reliable with the aid of a computerized system. First, the reliability of identification of the radiographic features providing the measures for classification improved by the computer-aided Cobb measurement method. In our previous study [10], the accuracy and reliability of the proposed Cobb measurement have been demonstrated. Second, the errors of judgment are reduced by using the computerized classification algorithm. With the aid of computer, the variability produced in the interpretation and memory of the classification criteria are avoided.

With the aid of computer, both intraobserver and interobserver repeatability are improved. The reliability is comparable to the method of Stokes and Aronsson [5] (average intraobserver $\kappa$ value of 0.90 versus 0.85 ). The comparison of the three trials suggests that reliability can be improved with an increased experience of using this computerized system. Although the proposed method still needs user interaction (i.e. to set the ROIs and to identify two sacral landmarks), only a few user skills are required. With the aid of computer, even an observer without clinical experiences (the observer 5) could achieve excellent intraobserver reliability of 0.93 . As the hardware requirement is only a personal computer and the software environment is Visual $\mathrm{C}++$, the developed software can be easily installed for clinical use.

The King classification is used to facilitate the objective assessment of scoliosis, i.e., to make it possible to "speak the same language" in the assessment. There is no gold standard for the classified results before surgery. Therefore, the accuracy of the system was evaluated by using the cases that were consistently classified by the surgeon three times without the computer aid. The comparison of the surgeon's first trails under the conditions with and without the computer aid confirmed the accuracy of the proposed method (95\% accuracy).

In the experiments, the pelvis and T1 to L5 vertebral levels were visible on each radiograph. Whether the quality of radiographs relates to the classification results needs further investigation. Computer-aided selection of fusion levels for scoliosis correction is also one of our future research directions.

\section{Conclusions}

The proposed computerized system may be valuable to assist a surgeon in the King classification of scoliosis. It can reduce the Cobb measurement variability and the human judgmental errors in the King 
classification. It can be used equally well by individuals with less clinical experience. This technique can be extended to assist other classification systems for scoliosis.

\section{Acknowledgements}

This work was supported in part by the grants from the Natural Science Foundation of China under Grants 61002049 and 61361010, and in part from the Key Program of the Yunnan Natural Science Foundation under Grant 2013FA008.

\section{References}

[1] Stokes IAF. Three-dimensional terminology of spinal deformity. A report presented to the scoliosis research society by the scoliosis research society working group on 3-D terminology of spinal deformity. Spine. 1994; 19(2): 236.

[2] King HA, Moe JH, Bradford DS, Winter RB. The selection of fusion levels in thoracic idiopathic scoliosis. J Bone Joint Surg Am. 1983; 65(9): 1302.

[3] Cummings RJ, Loveless EA, Campbell J, Samelson S, Mazur JM. Interobserver reliability and intraobserver reproducibility of the system of King et al. for the classification of adolescent idiopathic scoliosis. J Bone Joint Surg Am. 1998; 80(8): 1107.

[4] Lenke LG, Betz RR, Bridwell KH, Clements DH, Harms J, Lowe TG. Intraobserver and interobserver reliability of the classification of thoracic adolescent idiopathic scoliosis. J Bone Joint Surg Am. 1998; 80(8): 1097.

[5] Stokes IAF, Aronsson DD. Identifying sources of variability in scoliosis classification using a rule-based automated algorithm. Spine. 2002; 27(24): 2801.

[6] Stokes IAF, Aronsson DD. Computer-assisted algorithms improve reliability of King classification and cobb angle measurement of scoliosis. Spine. 2006; 31(6): 665.

[7] Lin H. Identification of spinal deformity classification with total curvature analysis and artificial neural network. IEEE Tran Biomed Eng. 2008; 55(1): 376.

[8] Poncet P, Dansereau J, Labelle H. Geometric torsion in idiopathic scoliosis: three-dimensional analysis and proposal for a new classification. Spine. 200; 26(20): 2235.

[9] Duong D, Cheriet F, Labelle H. Three-dimensional classification of spinal deformities using fuzzy clustering. Spine. 2006; 31(8): 923.

[10] Zhang J, Lou E, Shi X, Wang Y, Hill DL, Raso JV et al. A computer-aided Cobb angle measurement method and its reliability. J Spinal Disord Tech. 2010; 23(6): 383. 